\title{
Study on the Cultural Heritage and Three- Dimensional Simulation in the Design of City Cultural Square
}

\author{
Yu Xiao \\ Huanggang Normal University \\ Huanggang, China
}

\begin{abstract}
Based on the role of the historical development of Chinese cultural heritage in five thousand years in promoting the design of contemporary city square, this paper applies threedimensional simulation technology to design city square with Chinese traditional culture characteristics, integrates the Fengshui theory of the ancient traditional culture, overhanging eaves of buildings in ancient buildings, landscaping of the ancient garden aesthetics, etc. into the elements of modern city square design, and expresses in a new form, in the purpose of making people realize that Chinese ancient aesthetics elements have played an important role in contemporary natural ecological environment and cultural protection of the earth, and aiming to make people realize the importance of carrying forward the national culture essence and the urgency of the revitalization of Chinese civilization.
\end{abstract}

Keywords-three-dimensional simulation; city square; traditional culture; garden aesthetics

\section{INTRODUCTION}

Historical city square of China boasts the unique Chinese "square" style, different from city square of Europe. It evolves from "market" made up of crisscross streets with a long history dating back to the Southern Song Dynasty. With people gathering in a certain area to conduct various commercial trade activities, many buildings are built for the convenience of trade activity at that time. The business trading space formed spontaneously has the primary functional features of city square space and it can be called the rudiment of China's city square. With China's economic development, its city square development has been on the way all the time. As a space for entertainment and rest, city square has become an important part of urban residents' life and it is historical follow for China experiencing rapid economic growth since reform and opening-up. City square is a part of urban public art and an important part of the city, representing the image of city. Therefore, city square's design scheme emphasizes on embodiment of local regional culture and inheritance of cultural vein in order to create a space for residents to rest with a clear-cut beacon sign of local profound cultural heritage.

\section{LAYOUT DESIGN OF CITY SQUARE WITH FUSION OF Traditional Chinese CUlture}

Center principle is one of the basic principles emphasized most in Fengshui theory. According to historical record, as early as in pre-Qin period, the principle is stressed under the influence of Fengshui theory, promoting center position with a proper distance from the top, bottom, left and right. For example, the ancient city of Luoyang is the capital of Xia, Shang, Zhou, Han, Jin, the Northern Wei Dynasty, Sui, Tang and Liang because the governor prefers its center position. The capital of China's past dynasties is always in a center position for the convenience of controlling the country and balancing power and influence of all parties. At the primary stage of building Beijing city, the governor arranges a central axis to divide the whole plot to highlight the center position of the imperial city. Balanced arrangement is adopted on both sides of the axis and all downtown streets and facilities are arranged around the central city's axis.

At the primary stage of designing square, some changes are made to differentiate one square from another. Abiding by a series of principles in Fengshui theory, we design a square plane "Fig. 1" boasting the beauty of Chinese classic architecture and differing from other types of city square. Seeing from the plane, the square is seated facing north with four giant red diamonds supported by several fine wooden pillars, integrating into a whole with the central part of square and embodying characteristic principles emphasized in Fengshui theory and of ancient Chinese architectural design element, like cornice and brackets, city wall and courtyard brick wall, and garden water. "Conformal" is mentioned in Dazhuang Hexagram of the Book of Change, referring to adjusting measures to local conditions and the rehabilitating principle of returning to one's original nature based on surrounding environment. Square design embodies architectural elements of eaves tile, wall, courtyard house and water scenes of garden in ancient Chinese architecture in an abstract way, interpreting "conformal" in a different way. 


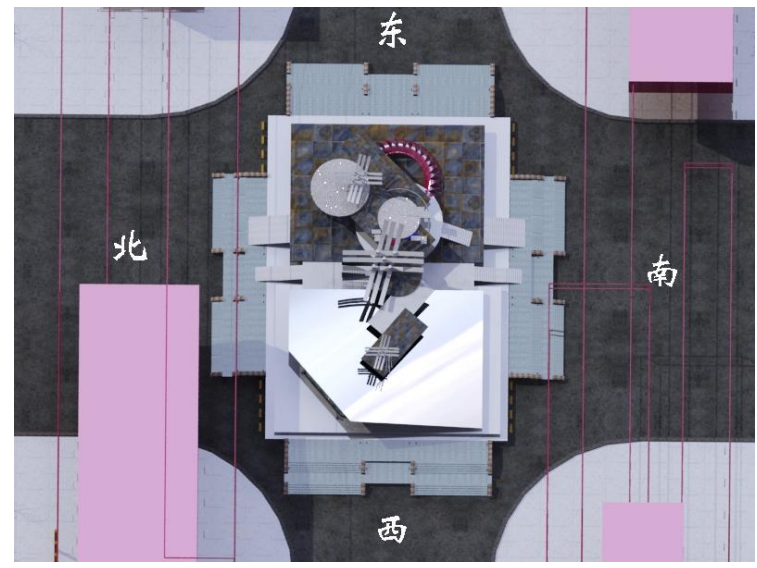

Fig. 1. Square plan

Traditional Chinese architecture is particular about round heaven and square earth. Temple of Heaven and Temple of Earth in Beijing are built according to the principle of round heaven and square earth in Ming and Qing Dynasties. Namely, Temple of Heaven is round and Temple of Earth is square. Ordinary people usually build a round pool in their square courtyard or dig a dome-shaped moon gate in the wall connecting two courtyards, embodying the concept of round heaven and square earth. In "Fig. 1", the stylobate in the center of the square is square and the zenith is round, conforming to ancient Chinese saying of "round heaven and square earth". Although the circular arc slate fails to cover the heaven and it is just an abstract metaphor here, the stylobate made of natural and plain stone and the thick mirror plane sun louver with gray tone bring out the best in each other.

Apart from simulating the nature in building according to the terrain and creating quiet twists and turns in the garden, Chinese gardens still abide by center position principle in Fengshui theory in layout. Overlooking plane layout of the whole garden, an axis extending from south to north is seated just in the middle. On the north side of the axis lie mountains and on the north side lies flat and open terrain. As to architecture surrounding the brook, there are pavilions, platforms, storied buildings and sheds on eastern and western sides.

\section{ARChitectural DeSign IN THE SQUare CENTER}

\section{A. Cornice Design in the Square Center}

In traditional Chinese architectural image, wooden rooftop is the most original and unique. In ancient architecture, girders and pillars and other wooden components form a wooden scheme structure with scattered heights, demonstrating the graceful top of slope. Besides, the cornice lightly turns up at the turning angle of the eave, boasting a significant symbol of ethnic elements of traditional and ancient Chinese architecture. This kind of entablature not only enlarges daylighting area inside the house, but also makes it beneficial to water draining and defending against snow. At the first sight, it looks like a roc wing taking off all around the architecture, making the ancient architecture with wooden structure lighter, handier and more vigorous shown in "Fig. 2".

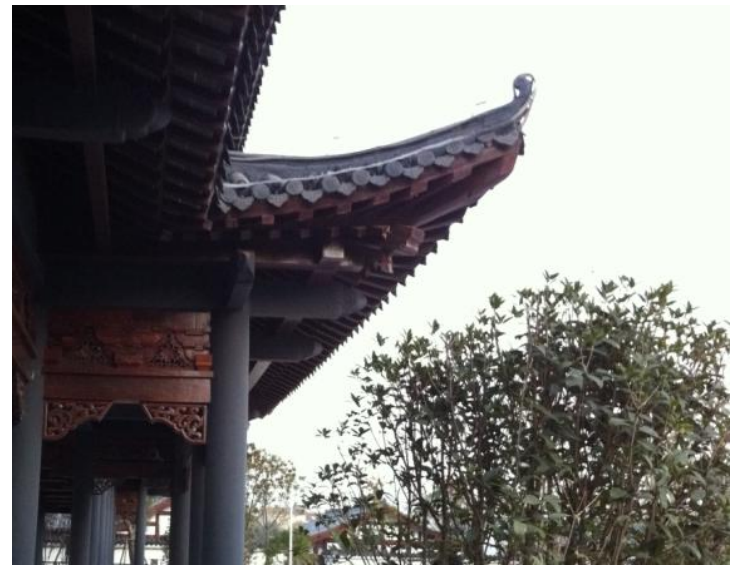

Fig. 2. Cornice in Chinese ancient architecture

Three buildings made of polished white marble and supported by sturdy uprights cross in the center of the square, with cornice flying to the end of the heaven. The abstract image here represents the ancient cornice "Fig. 3" and "Fig. 4" in Chinese classic architecture with upwarping entablature. The flying gesture is shaped like flying birds spreading out their wings in a vivacious and light way.

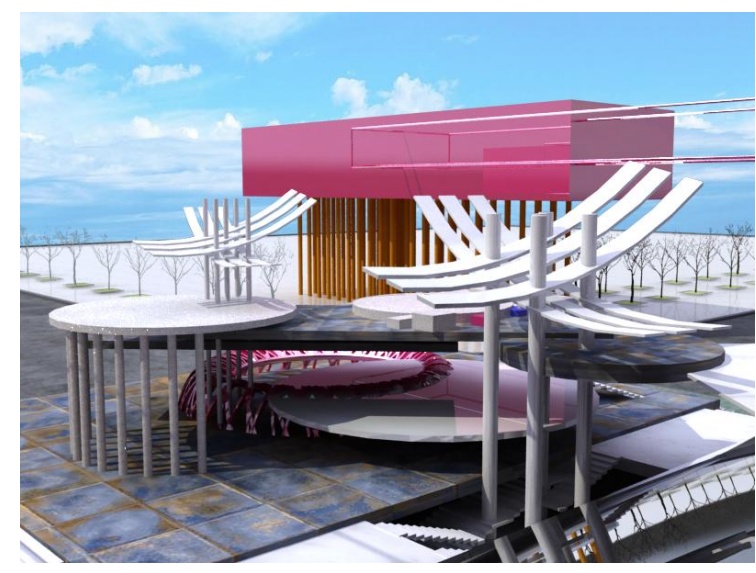

Fig. 3. Cornice sculpture in the square center

\section{B. Design of Bird Nest in the Square Center}

Influenced by the bird nest building as the main venue of 2008 Beijing Olympic Games, there is a red steel bird nest frame, a round rooftop, stone aisle columns and sun louvers "Fig. 4" in the eastern center of the square, representing contemporary architectural achievement. People enter the square and ascend step by step and look up at the sky under the iron frame of the steel bird nest, looking forward to realize a certain dream as early as possible. If every dream comes lightly in such an enclosed tranquil environment, every step to realize the dream must be striven in the soup, such enlightenment brought to viewers by each piece of steel of this hope nest. 


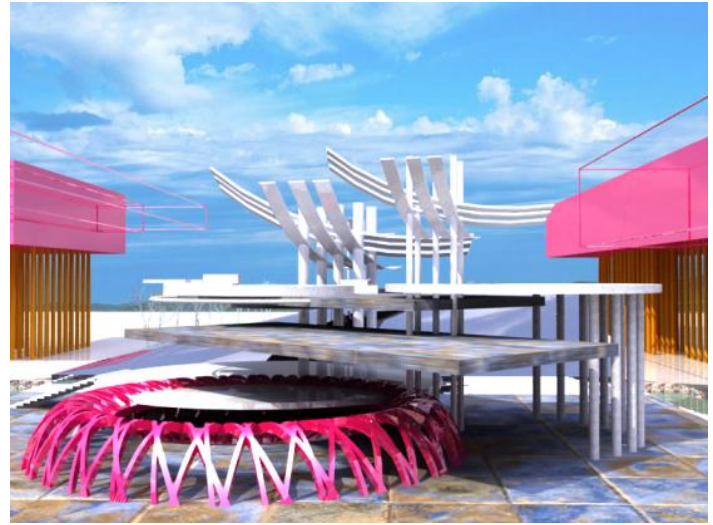

Fig. 4. Design sketch of bird nest and cornice in the square center

\section{C. "Rammed Earth Platform" at the Center Bottom of the Square}

The center bottom of the square is built with reference to rammed earth platform in ancient Chinese architecture. A number of small single buildings are integrated on a "rammed earth platform" "Fig. 1" to achieve the architectural style of large volume and changeable form. This kind of building owns a high position with grand appearance. Giant natural stone panel is placed on "rammed earth platform" and stairs connect the platform with floor slab. The whole giant natural stone panel can be regarded as a square as well as a giant sculpture. People can "climb" onto the sculpture to look into the distance or walk onto the square to overlook "Fig. 5". The actual space is limited while the artistic conception is infinitely profound and lasting. Various and abundant bardian landscapes and implicit and endless conception are created in the limited space. Besides grasping at macro level, the whole space should be divided into large, medium and small levels. As the result of landscape division, each independent environmental space has its own unique theme, forming a harmonious atmosphere of different artistic conceptions.

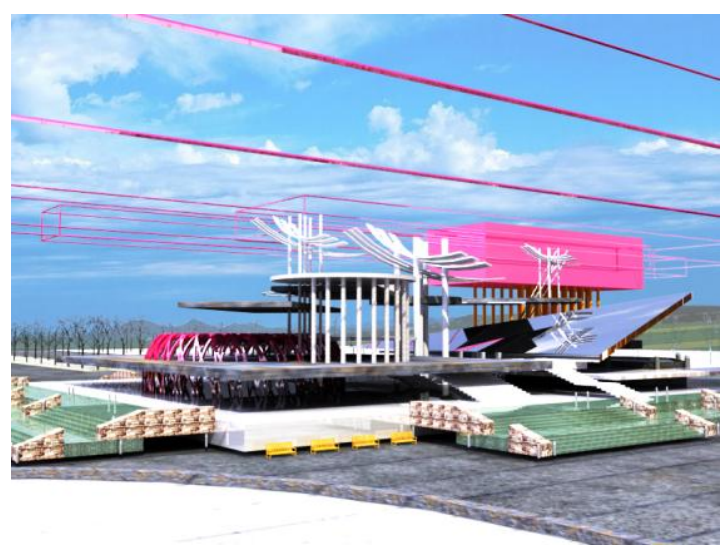

Fig. 5. "Rammed earth platform" in the square center

\section{Application of "Kui Bi" Technique in Design of Architecture in the Square Center}

"Kui Bi" contains two relevant and contrasted concepts. "Bi" means screening people's sight and making it not able to pass through. "Kui" is the opposite of "Bi", referring to transmission of unsheltered sight. Therefore, "Kui Bi" means both penetrable and impenetrable, and sheltered and unsheltered, forming an artistic atmosphere with fictitious and realistic comparison and complementation of hide and reveal. "Depth of field" refers to depth of front scenery "Fig. 6" seen by naked eyes and based on aesthetic feelings and aesthetic imagination. The depth is faint and vague rather than apparent. If the front scenery is partly permeable and partly sheltered, its subsequent scenery must be partly hidden and partly visible, giving people a strong vertical feeling arousing further exploration along with the sense of beauty.

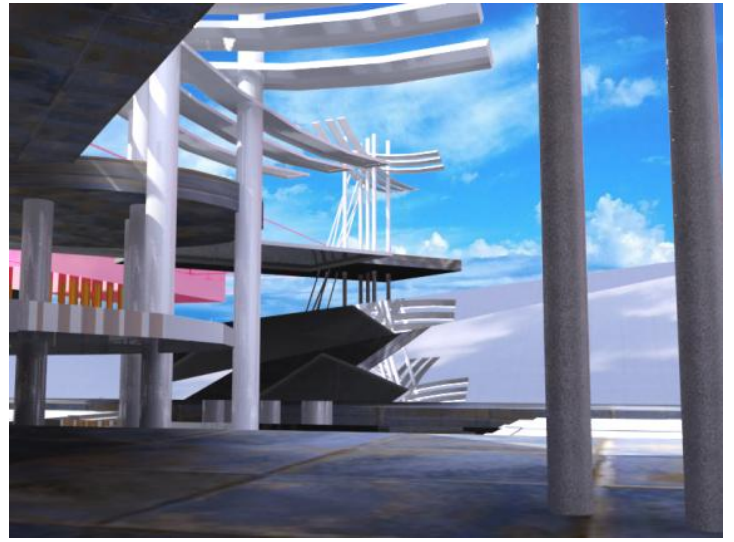

Fig. 6. Visual "depth of scenery" of square center from the east to the west

The square design adopts the technique of "partly Kui and partly $\mathrm{Bi}$ " in "Kui Bi" with a main structural function of achieving unification in hidden and visible architectural scenery to create the beauty of deep interest and charm, which is the theory of "both hidden and visible". Five tall and sturdy uprights "Fig. 7" are inserted among stairs in the internal center of the square, whose design inspiration comes from beam column in ancient Chinese architecture. Since stairs connect the upstairs and downstairs in a straightforward way, these five uprights greatly enrich sense of depth of space connection, boasting the artistic conception of "both hidden and visible" in Chinese classical gardening. The space of square center is not that widely-open and several twists and turns must be taken to achieve open and clear, like loss of depth of field.

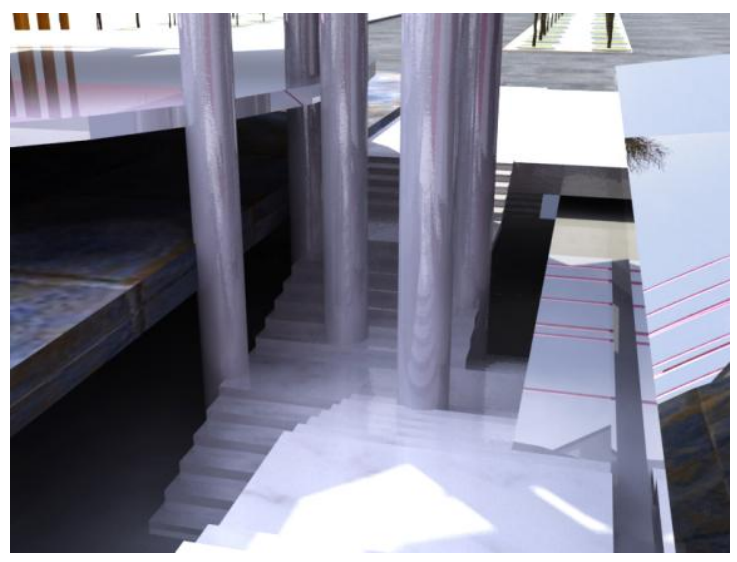

Fig. 7. Five uprights enrich the sense of arrangement of spatial connection 


\section{E. Design Method of "Broad View" and "Far-Reaching Mood"}

Besides the creation technique of "Kui Bi", two phrases appear in a good deal of records of garden in ancient China: "broad view" and "far-reaching mood", referring to two different spacial concepts. "Broad view" refers to winding, shelter and deep turn, like placing oneself on intermountain hills, and hiding oneself in shrubs and a rank growth of grass with restrained environment and tortuous space. "Far-reaching mood" refers to emptiness and loftiness with boundless sight and distant space.

Scattered and dense layout boasts insubstantial scene and real scene with free openness. "The bamboo path extends to a secluded quiet place and the meditation abode is accompanied by lofty bamboo groves". The "bamboo path" in this famous saying is changed into "winding path" as a result of modern aesthetic and the main choice for tour route. The circular arc slate in the center of the square redefines the connotation of a winding path leading to a secluded quiet place "Fig. 8". Ignoring surrounding environment, people can get up on the round slate platform and go along the circular arc touring route When raising the head to look at round arcs overhead, people comprehend the deep meaning of "round heaven and square earth" unconsciously.

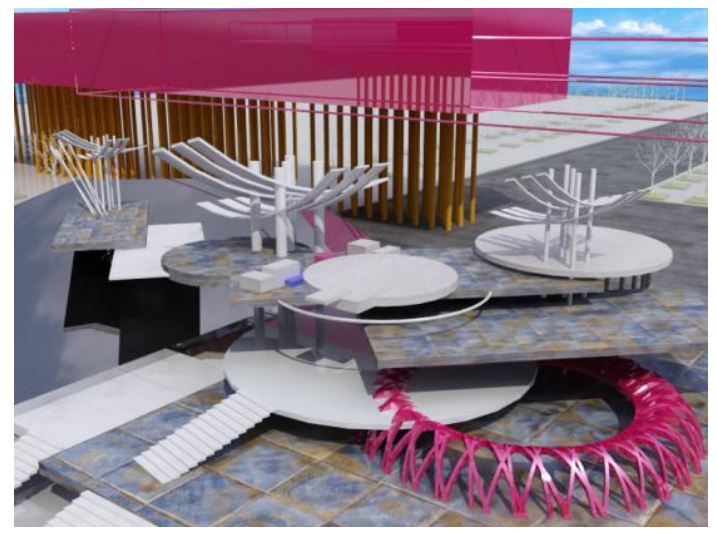

Fig. 8. The circular arc slate redefines the winding path leading to a secluded quiet place

Design of square center imitates artistic presentation techniques of "broad view" and "far-reaching mood" in garden construction and the designed space is organically divided into layered small and large parts by three-dimensional simulation technology "Fig. 9". The open area of the space provides a secluded long and narrow winding region and a deep winding broad atmosphere with boundless sight and distant farreaching mood to achieve the aesthetic feeling of coexistence of broad view and far-reaching mood and ingenuity of double replacement. If what people see during their travel is exactly the same as they expect, they will feel bored and insipid. Therefore, part of what people desire to see needs to be sheltered.

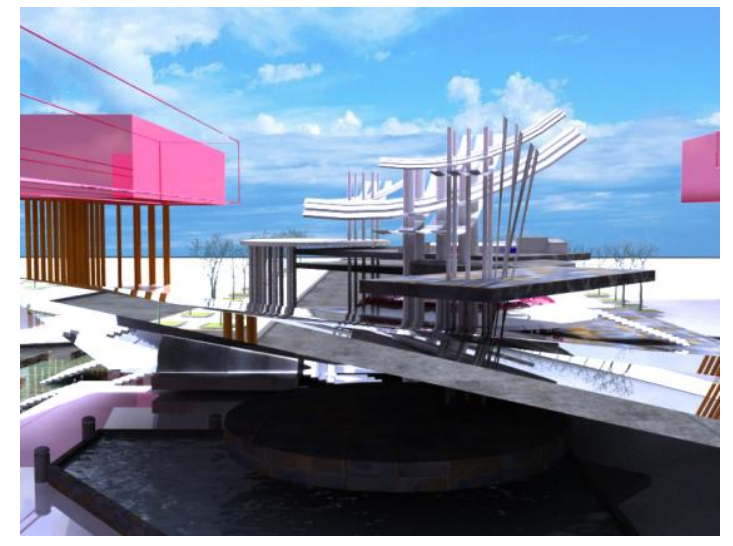

Fig. 9. Spatial division of square center

\section{FACADE DESIGN OF THE SQUARE}

\section{A. Facade Design Sketch of West Square}

Chinese classical garden boasts an open and clear layout, pure scenery, clear-cut ups and downs in outline, brightness of the sky and shadow of the clouds, and thin timber piles supporting the giant building. Seeing from the outside to the inside, the internal scene is sometimes virtual and sometimes real. In Chinese ancient architecture, scenery of inner courtyard is always sheltered by wall doors with a little effused "emerald green" in flirtatious expression. The appearance of the whole square center can not be directly viewed from the west main entrance "Fig. 10" and the prying curiosity can only be satisfied through aisle columns layer upon layer and chunky red gate. Contrary to the ancient saying of "entering the gate and seeing the mountain", landscape in the garden is sheltered to prevent visitors from seeing all at one glance. It is an artistic technique of control and repression in advance for the purpose of promotion in order to firstly ensure that the quiet and beautiful environment inside the garden is not disturbed by the outside world and in order to secondly eradicate the necessity of opening the door to see the scenery layer upon layer. For example, go past the object standing in the way and dense willow trees and bright flowers come into eyes.

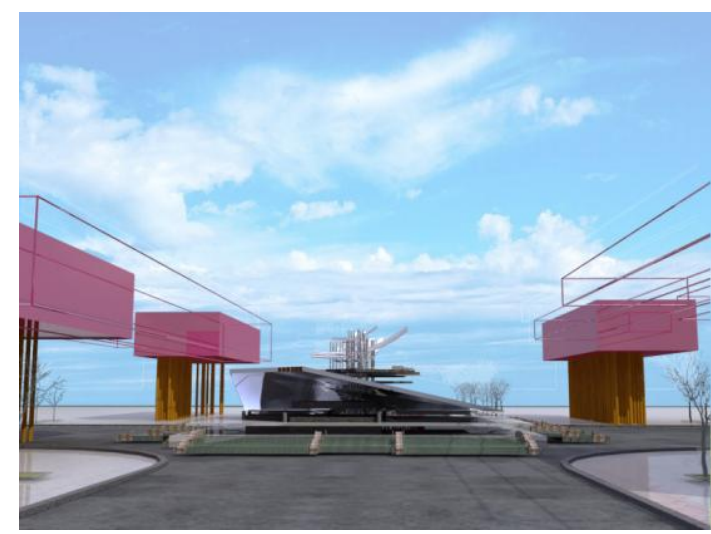

Fig. 10. Facade design sketch of west square 


\section{B. Facade Design Sketch of South Square}

Contrary to visual effect of he west, seeing from the south, displayed objects lie directly before people's eyes with a panoramic view of square center's main appearance, conforming to the saying of "entering the gate and seeing the mountain" in ancient garden construction "Fig. 11".

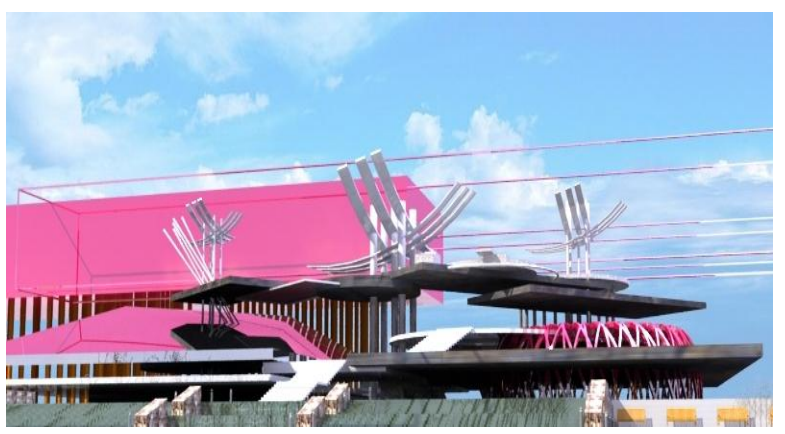

Fig. 11. Facade design sketch of south square

Two giant red sculptures stand in the latitudinal direction of the square and their mirror face can reflect color of the sky, respectively representing the front and back door wall of Chinese ancient architecture. Purity of red is lowered and the rose-bengal tone and hope nest in the center of the square add radiance and beauty to each other.

\section{CONCLUSION}

It is a detail drawing of the square built based on the above described contents. Scenes are created by use of lamplight, material, chartlet and rendering and computer model is created based on simulation of reality scene. 3DMAX can not only change the model's design pattern at any time, but also can create more complex design works than in reality and show it out in an unimaginable real way. The three-dimensional simulation technology helps designers get rid of the tedious and inefficient design method, reduces time of the process from primary conception to modeling, rendering and the final accomplishment of the drawing, materializes designers' ideas, enhances designers' work efficiency and enables designers to express their thought in a more straightforward and clear-cut way.

\section{REFERENCES}

[1] Xiao Yu. Application of Computer Three-dimensional Simulation in City Square Design and Research on It. Master Thesis of Wuhan University, 2011

[2] Xie Lisheng, Ge Jun, Mi Xinwu. Research on Mountain Planning of Cities and Towns in Jiangchuan of Yunnan, Construction, Structure and Environmental Engineering International Academic Conference. October 20 to 21, 2012, Yichang, China, www.gccsee.org

[3] Wen Zeng. City Square Design, Liaoning Fine Arts Publishing House, The Third Edition, 2008

[4] Cai Yongjie. City Square: Historical Context, Impetus of Development and Space Quality, Southeast university press, 2006. Chinese Library Classification Number TU Building Science 